Bull. Mater. Sci., Vol. 2, Number 4, November 1980, pp. 251-264. (C) Printed in India.

\title{
Studies on thick films of photoconducting cadmium sulphide*
}

\author{
D P AMALNERKAR, M S SETTY, (Miss) $N$ R PAVASKAR and \\ A P B SINHA \\ Physical Chemistry Division, National Chemical Laboratory, Poona 411 008, India \\ MS received 15 May 1980
}

\begin{abstract}
Highly photosensitive films of CdS have been prepared using the thick film technique. The films obtained from the composition containing CdS -100 , $\mathrm{CdCl}_{2}-10$ and $\mathrm{CuCl}_{2}-0.05$ parts by weight (reacted at $500^{\circ} \mathrm{C}$ ) are found to give the best photosensitivity on firing at $600^{\circ} \mathrm{C}$. The ratio of light to dark current $\sim 10^{8}-10^{9}$ which is considerably higher than what is reported for thin films, single crystals and sintered layers. A strong chemisorption of oxygen is found to be responsible for high photosensitivity. The spectral response for doped CdS film is similar to that obtained for thin films, single crystals and sintered layers and also shows a red shift with increasing $\mathrm{Cu}$ concentration. However, the undoped $\mathrm{CdS}$ has a broad spectral response at room temperature ranging from $\lambda=550$ to $690 \mathrm{~nm}$; unlike the thin films and single crystals which give a sharp peak at $\lambda=$ 510 to $520 \mathrm{~nm}$. A probable explanation has been suggested for this type of behaviour.
\end{abstract}

Keywords. Cadmium sulphide; thick film technique; photosensitivity; spectral response.

\section{Introduction}

CdS photoconductive films have been studied intensively in the past 25 years in view of their potential applications in various electronic devices. Studies of the CdS films prepared by various methods such as sintering (Thomsen and Bube 1955; Thomas and Zdanuk 1959), vacuum deposition (Veith 1950, 1955), chemical deposition (Pavaskar et al 1977; Sathaye and Sinha 1976) and thick film technique (Nicoll and Kazan 1955; Micheletti and Mark 1968) have been reported. However, there is not much literature on the photoconducting properties of CdS films prepared by the thick film technique which is essentially a screen-printing and firing process. Nicoll and Kazan (1955) had earlier used the process of screen-priating to prepare powdered layers of photoconducting CdS. Micheletti and Mark (1968) have demonstrated and explained substantially the consequences of the oxygen chemisorption on the equilibrium conductivity and on the transient photoconductivity of sintered CdS layers prepared by this technique. However, the photosensitivity (i.e. light to dark current ratio) generally obtained has been upto $10^{7}$ in all the methods mentioned earlier. In the course of the present work, photoconducting films with much higher photosensitivity $\sim 10^{9}$ have been obtained

* NCL Communication No. 2502. 
by this technique. It is the purpose of this paper to present the detailed preparative procedure and the photoconducting properties of such films.

\section{Experimental}

\subsection{Preparation}

Preparation procedure, in general, can be described in three steps :

\section{(i) Activation or doping of pure CdS}

Highly pure CdS (99.999\%) made by Koch-Light and Company was used as the starting material for this work. For activation, $\mathrm{CdS}$ was mixed with $\mathrm{CdCl}_{2}, 2 \frac{1}{2}$ $\mathrm{H}_{2} \mathrm{O}(10 \%$ by weight of $\mathrm{CdS})$ which acted as a flux and $\mathrm{CuCl}_{2}, 2 \mathrm{H}_{2} \mathrm{O}(0.05$ to $1 \%$ by weight of $\mathrm{CdS}$ ) which acted as an activator.

Mixtures containing the above mentioned components were ball-milled for $2 \mathrm{hr}$ and then subjected to the initial bulk-firing at $500^{\circ} \mathrm{C}$ for $2 \mathrm{hr}$ in air. This initial firing achieves the preliminary distribution of activator components into the $\mathrm{CdS}$ matrix. The resulting mass in the form of brownish lumps was thoroughly milled using double distilled acetone to ensure a sufficiently fine particle size.

\section{(ii) Formulation of the paste}

The paste was formulated by mixing the activated $\mathrm{CdS}: \mathrm{Cu}: \mathrm{Cl}$ powder with a solution of ethyl cellulose in a mixture of solvents consisting of butyl cellosolve, butyl carbitol acetate and terpineol in the proportion $30: 30: 40$. Fluidity of the paste depends upon the extent of organic part which goes in its formulation (i.e., solid to liquid ratio) (Setty 1979). The ratio of activated CdS powder to the solution of ethyl cellulose binder in a solvent mixture was kept at 75:25 in formulating the paste. This particular ratio for the paste formulation was found to be thixotropic in nature and gave good line definition of the prints.

\section{(iii) Screen-printing and firing}

Silk-bolting cloth (160 mesh), with appropriate apertures blocked to form the desired pattern, mounted on a rigid frame was used as the screen. Screen-printing was accomplished by a flexible squeegee stroking the paste across the screen surface onto ceramic alumina substrates. The wet films obtained in this way were dried at $100^{\circ} \mathrm{C}$ for $20 \mathrm{~min}$ and fired afterwards at the desired temperature for $10 \mathrm{~min}$ in oxygen-free nitrogen atmosphere. A specially designed silica envelope was used for this purpose. This high temperature firing matures the thick film elements and bonds them integrally to the substrates. Thick films of CdS obtained in this way ware found to be well-adherent to the substrates. Using this technique it is possible to fabricate photoconductive films of CdS with any arbitrary area and desired photoconducting characteristics.

\subsection{Preparation of test samples}

For investigating the effect of preparation parameters particularly firing temperature and impurity concentration on the photoconducting properties of these films, 
a step-wise variation of these parameters at each part of the process was conducted. To prepare test samples for this purpose, (i) variation in firing temperature of the films was carried out from 400 to $800^{\circ} \mathrm{C}$ for one pilot formulation of the paste and (ii) variation in $\mathrm{Cu}$ content of the films was carried out from 0.05 to $1 \%$ by wt. of CdS. Details of the preparation were the same as described in 2.1. In addition to this, thick films of pure CdS were also processed.

\subsection{Electrode materials}

As indium was reported to make good ohmic contact with CdS, it was evaporated in vacuum $\left(10^{-5} \mathrm{~mm}\right.$ of $\left.\mathrm{Hg}\right)$ onto masked thick film samples. Silver was then vacuum-deposited over indium to enable easy soldering of the lead wires. Leads of 46 gauge silver-copper wires were soldered directly to the silver deposit.

\subsection{Techniques and methods of measurements}

The dependence of photosensitivity on preparation conditions, current-voltage characteristics, photocurrent-intensity behaviour and the spectral response of these films were studied.

Dark current measurements were carried out with electrometer amplifiersTrombay EA 810 and EA 814 of ECIL. In view of the very high dark resistance of these films, special precautions had to be taken in earthing the equipment to avoid pick-up of a.c. signals which could otherwise lead to spurious responses. Measurements in dark were performed by mounting the samples in a thermally insulated and shielded dark box.

Measurements in light were done by illuminating the samples with $500 \mathrm{~W}$ projection lamp. Electrometer amplifiers and Philips VTVM (GM 6009/90) were used for this purpose. To establish current-voltage relationships, a d.c. regulated variable voltage power supply (Aplab Model 7122) was used.

The intensity of illumination was varied using neutral density filters and measured with a Lux-meter (Aplab type ML 4420).

For spectral response determination the sample was mounted in a specially constructed all-metal vacuum cryostat having a window through which the sample could be illuminated. A Hilger monochromator with a $500 \mathrm{~W}$ projection lamp was used as a light-source for these measurements. For photocurrent measurements, electrical connections to the sample were made through a 9-pinglass-metal vacuum lead-through. These measurements were conducted at $300^{\circ} \mathrm{K}$ and $93^{\circ} \mathrm{K}$.

All current measurements (in dark and in light) except those involved in spectral response determination were performed with a d.c. applied potential of $60 \mathrm{~V}$ while those in spectral response determination were carried out with a d.c. applied potential of $30 \mathrm{~V}$.

\section{Results}

\subsection{Effect of preparation conditions on photosensitivity}

The effect of firing temperature on the dark current and the photocurrent is illustrated in figure 1. These two curves reveal that both the dark current and the 


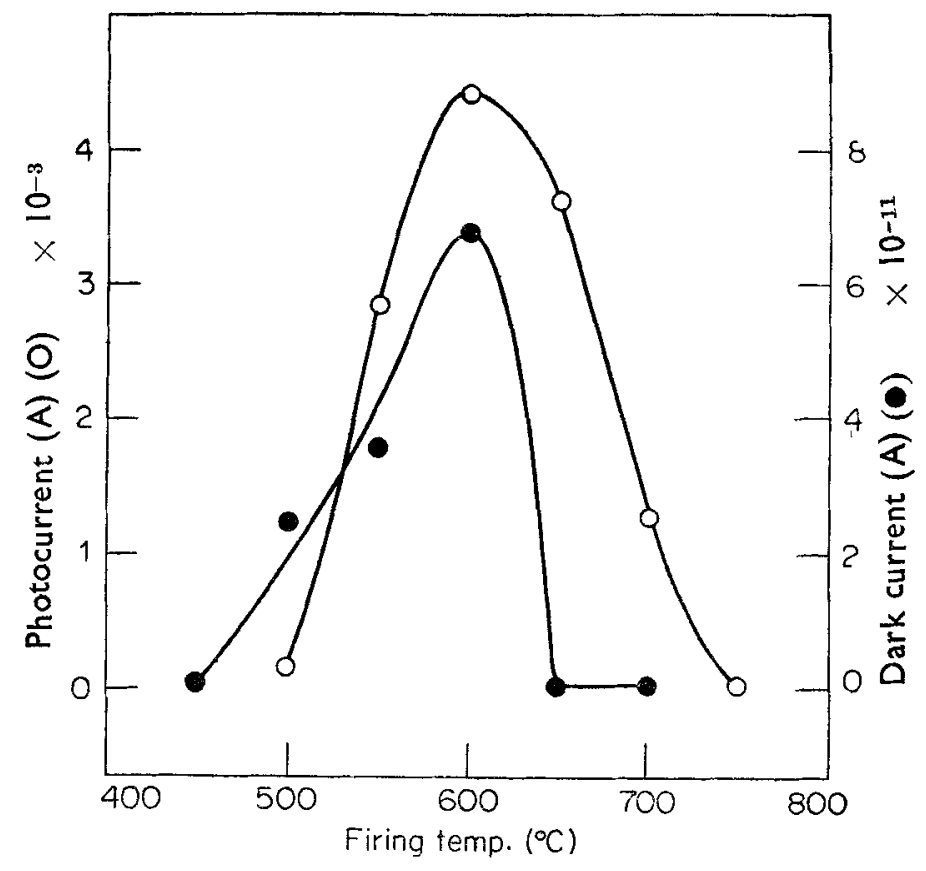

Figure 1. Effect of firing temperature on the photoconducting characteristics of CdS thick films. (Copper concentration $=0.05 \%$ by wt.)

photocurrent increase with increase in the firing temperature upto $600^{\circ} \mathrm{C}$ and decrease afterwards with further increase in the temperature. It is also noticed that adhereace of the film to the substrate becomes poorer with an increase in the firing temperature above $600^{\circ} \mathrm{C}$. It is found that photosensitivity (defined as light to dark curreat ratio) is the highest for the films fired at $650^{\circ} \mathrm{C}$. However, because of the adherence problem at this temperature, firing at $600^{\circ} \mathrm{C}$ is preferred.

The effect of $\mathrm{Cu}$ conceatration on the photocurrent of these films is plotted in figure 2 which shows a decrease in photocurrent with increase in $\mathrm{Cu}$ concentration. However, it has been observed that these films do not show an appreciable change in the dark current with increase in $\mathrm{Cu}$ concentration.

These observations have resulted in adopting an optimum firing temperature of $600^{\circ} \mathrm{C}$ and optimum copper concentration $-0.05 \%$ added as copper chloride by wt of CdS to give the superior and reproducible photosensitivity.

\subsection{Current-voltage characteristics}

Current-voltage characteristics of the undoped, i.e., pure CdS thick films, in dark and in light, are plotted in figure 3 . These plots show a slight deviation from the linearity indicating a departure from the perfect ohmic behaviour.

In figure 4 current-voltage characteristics of doped thick films with optimum $\mathrm{Cu}$ concentration are presented. The dark current as well as the photocurrent varies linearly with the appliea voltage. This ohmic dependence of the current 


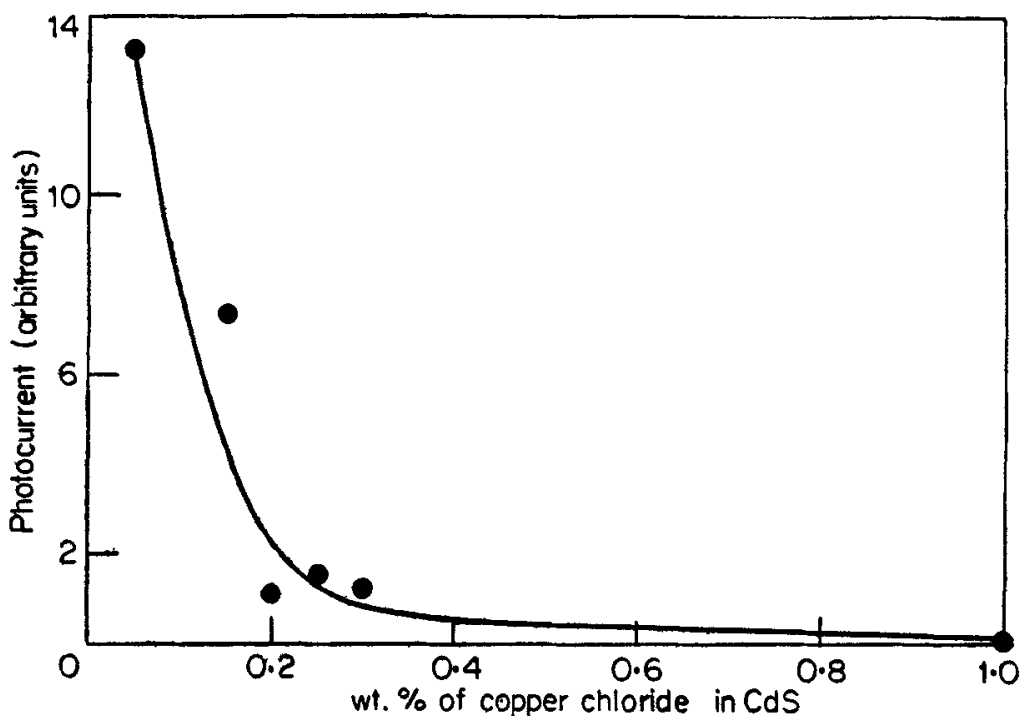

Figure 2. Effect of copper concentration on the photocurrent of CdS thick films, at room temperature and under constant illumination (Firing temperature $=600^{\circ} \mathrm{C}$ ).

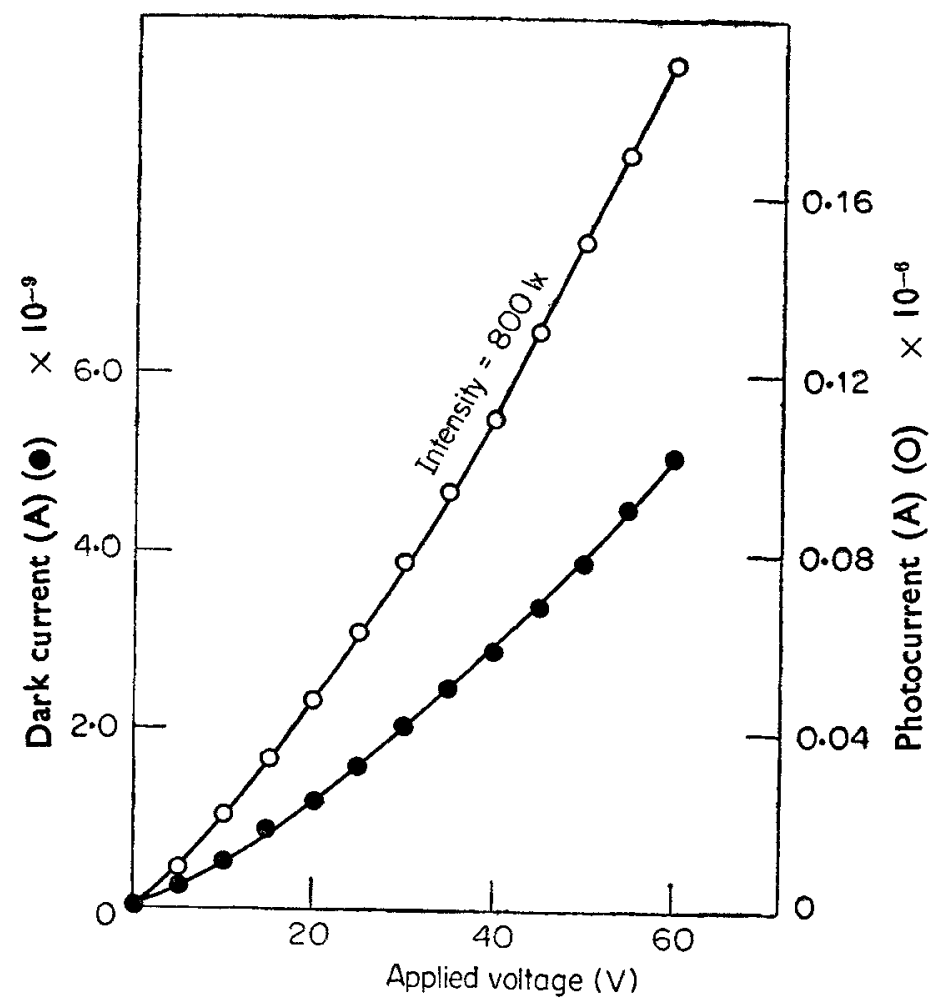

Figure 3. Current-voltage characteristics of undoped CdS thick film (At room temperature). 


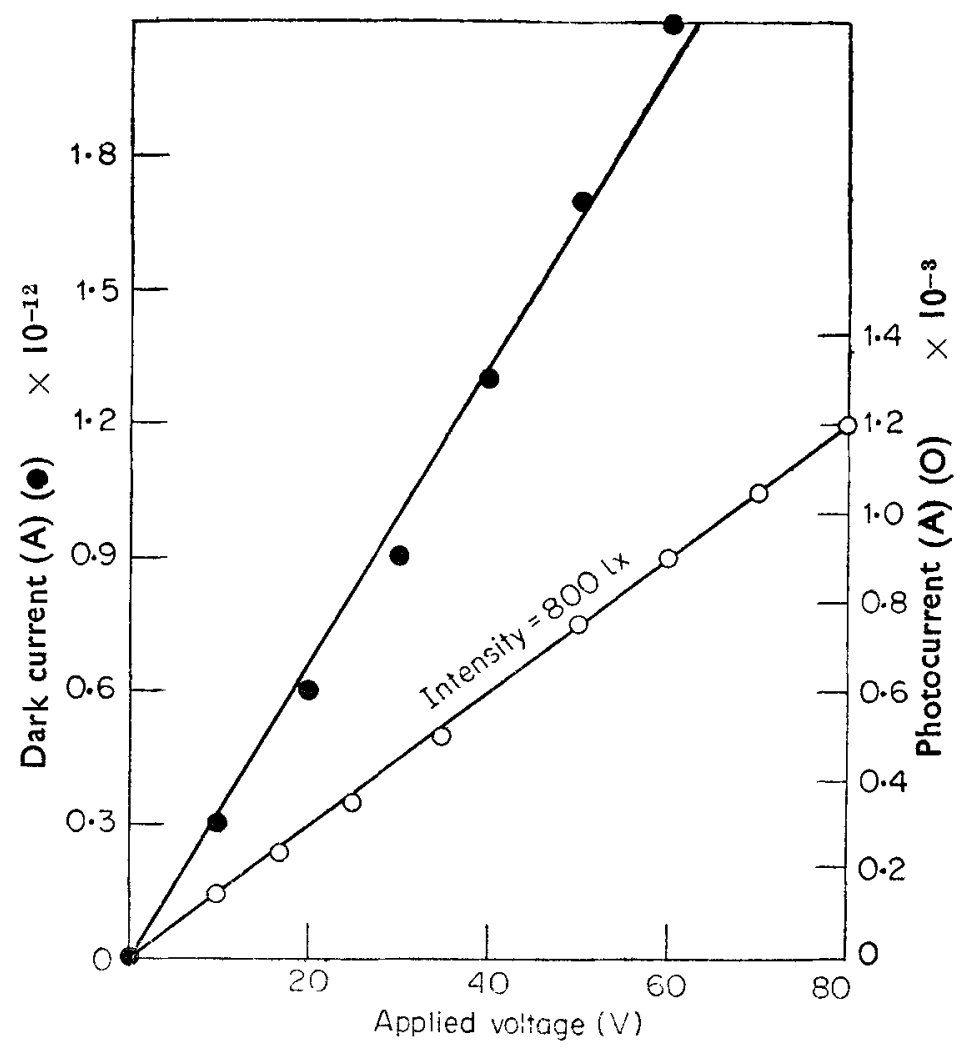

Figure 4. Current-voltage characteristics of doped CdS thick film. (At room temperature).

on the applied voltage is also confirmed with doped thick films of other $\mathrm{Cu}$ concentrations.

\subsection{Dependence of photocurrent on the intensity of illumination}

This dependence is plotted in figures $5 \mathrm{a}$ and $5 \mathrm{~b}$. These plots indicate that photocurrent varies as $I^{n}$ for these films, where $I$ is the intensity of illumination and (a) $n=0.5$ for pure CdS films, (b) $n=0.5$ (for lower intensities) and $n=1.0$ (for higher intensities) for the doped films with lower copper concentration (viz., 0.05 to $0.1 \%$ by wt), (c) $n=1.8$ for doped films with higher copper concentration (viz., $0 \cdot 3$ to $1 \cdot 0 \%$ by wt.).

\subsection{Spectral response measurements}

Spectral response curves associated with undoped thick film (at $300^{\circ} \mathrm{K}$ and $93^{\circ} \mathrm{K}$ ) are depicted in figure 6 . At $300^{\circ} \mathrm{K}$, this curve shows a response peak in the region of $\lambda=555 \mathrm{~nm}$ which is followed by a fairly constant response upto $\lambda=690 \mathrm{~nm}$. However, the spectral response curve at $93^{\circ} \mathrm{K}$ of the same sample indicates a sharp response peak at $\lambda=515 \mathrm{~nm}$ with a substantial decrease in the magnitude of photocurrent. 


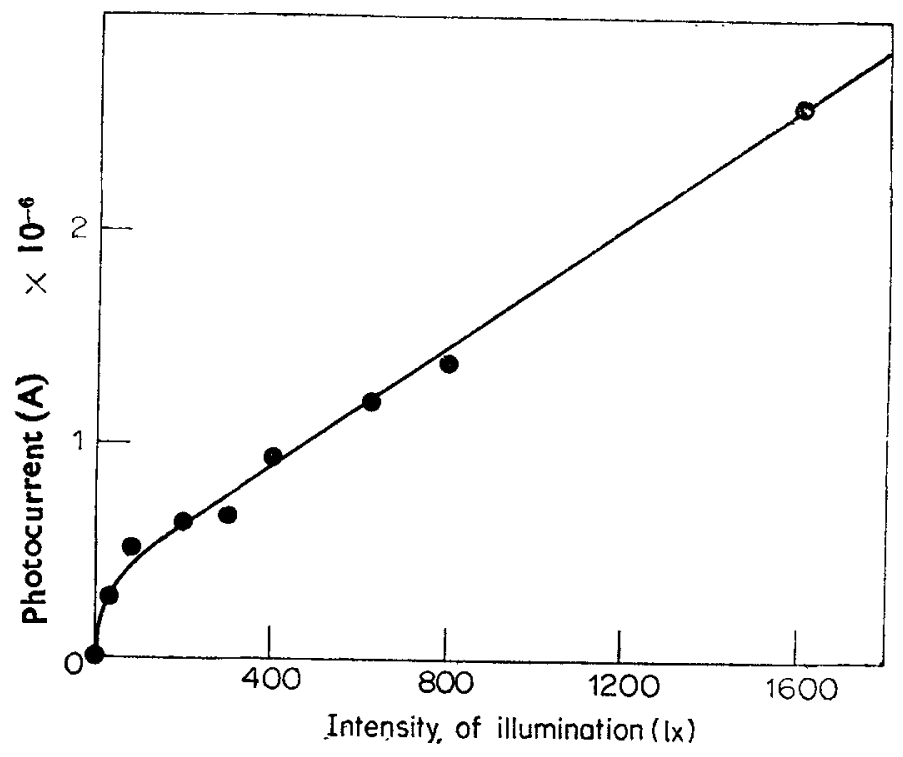

Figure 5 a. Variation in photocurrent with intensity of illumination for undoped thick film.

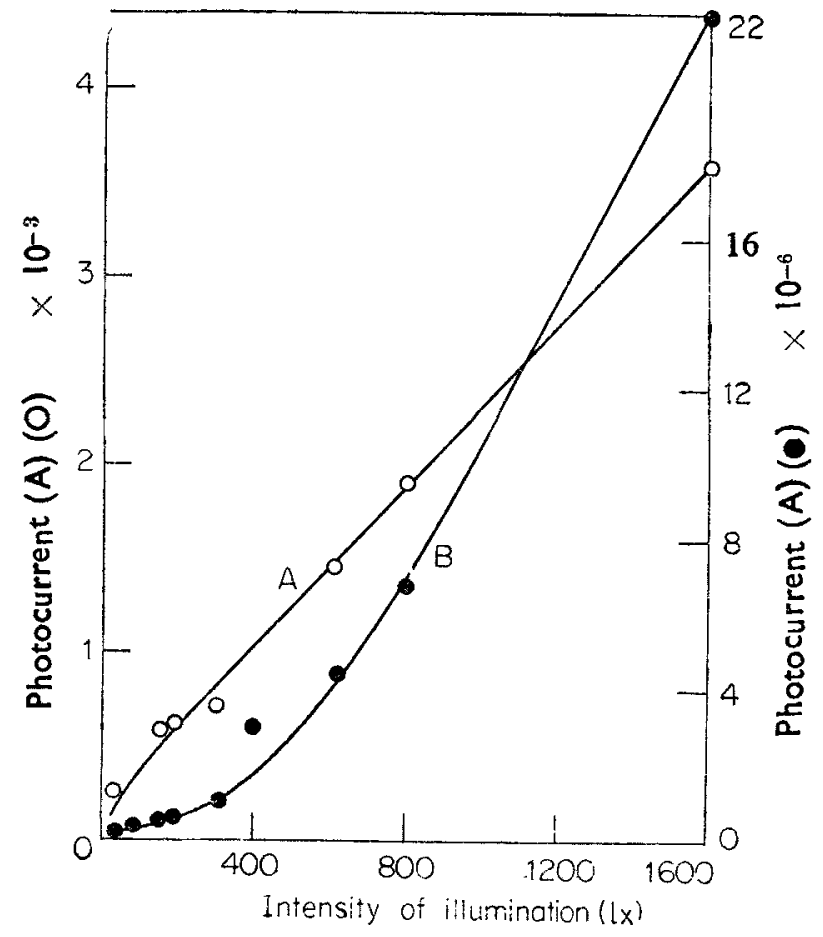

Figure 5b. Variation in photocurrent with the intensity of illumination of doped thick films, $\mathrm{A}-$ Lower $\mathrm{Cu}$ conc. $(0.05 \%$ by wt), B-Higher $\mathrm{Cu}$ conc. $(0.3 \%$ by wt). 


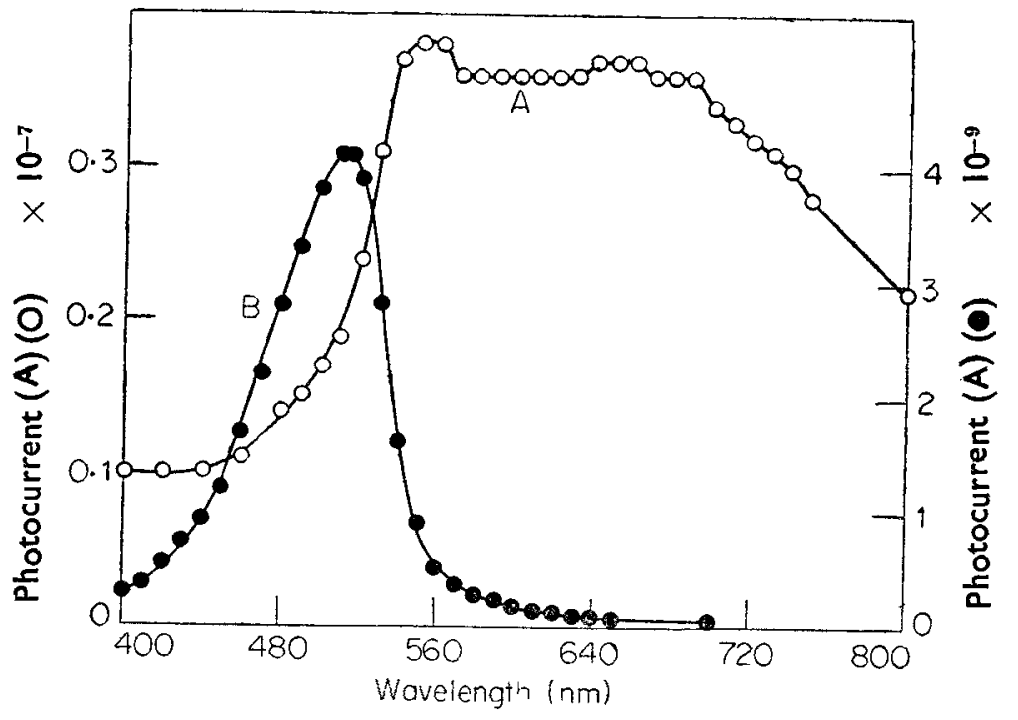

Figure 6. Spectral response for undoped CdS thick film. (Curve $A-300^{\circ} \mathrm{K}$, curve $\left.\mathrm{B}-93^{\circ} \mathrm{K}\right)$.

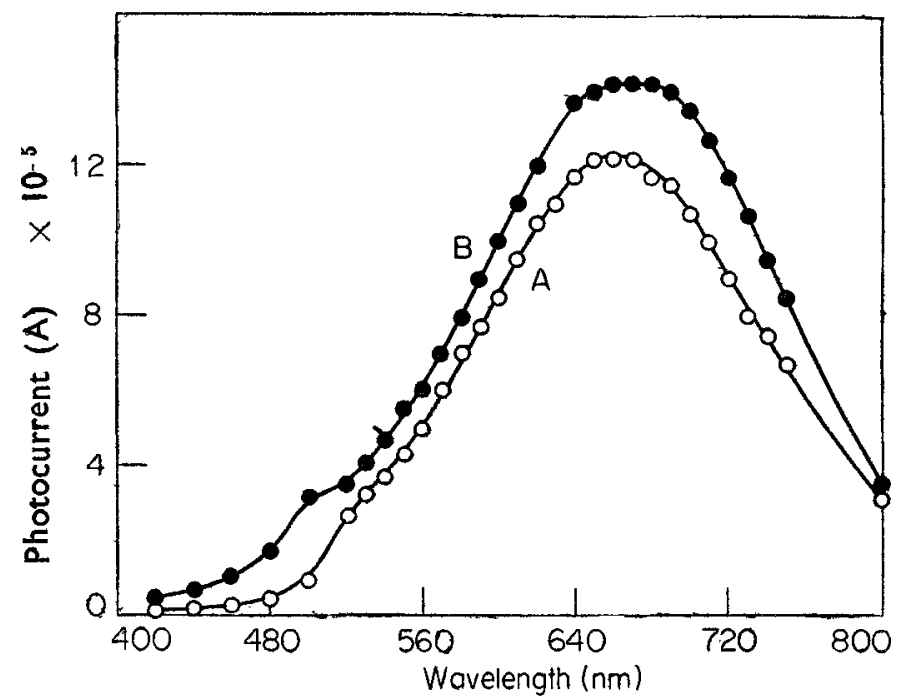

Figure 7. Spectral response for doped CdS thick film with optimum copper concentration. (Curve $\mathrm{A}-300^{\circ} \mathrm{K}$, Curve $\mathrm{B}-93^{\circ} \mathrm{K}$ ).

Spectral response curves of the doped thick film with optimum copper concentration (i.e. $0.05 \%$ by wt) at $300^{\circ} \mathrm{K}$ and $93^{\circ} \mathrm{K}$ are presented in figure 7 . The curve at $300^{\circ} \mathrm{K}$ shows a relatively broad response peak in the region of $\lambda=660 \mathrm{~nm}$. At $93^{\circ} \mathrm{K}$ the response peak remains unchanged with a little increase in the magnitude of photocurrent. 


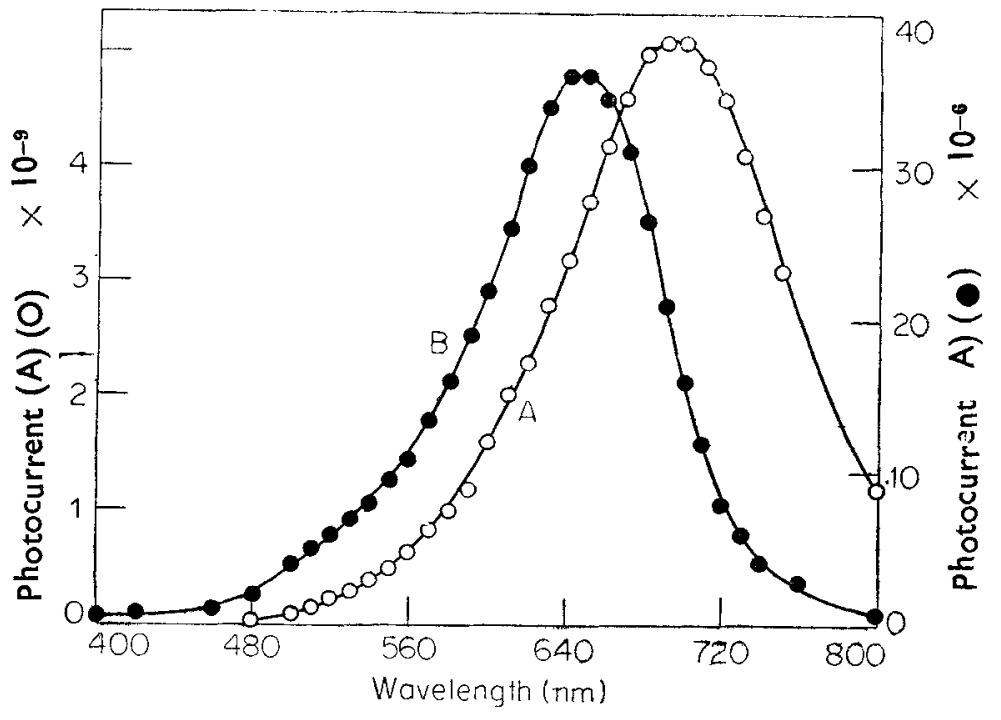

Figure 8. Spectral response for heavily doped $\mathrm{CdS}$ thick film. (Curve $\mathrm{A}-300^{\circ} \mathrm{K}$, Curve $\left.\mathrm{B}-93^{\circ} \mathrm{K}\right)$.

In heavily doped thick films ( $\mathrm{Cu}$ concentration more than $0 \cdot 25 \%$ by wt), a fairly sharp response peak is observed in the region of $\lambda=690 \mathrm{~nm}$ at $300^{\circ} \mathrm{K}$ (figure 8). But at $93^{\circ} \mathrm{K}$ the response peak shows a blue shift to $\lambda=650 \mathrm{~nm}$ in this case, with a drastic rise in the magnitude of photocurient from $10^{-9}$ to $10^{-5} \mathrm{~A}$.

It is also noticed that in doped thick films of CdS with the medium copper concentration (ranging from $0 \cdot 1$ to $0 \cdot 25 \%$ by wt), a fairly sharp response peak occurs in the region of $\lambda=645 \mathrm{~nm}$ at $300^{\circ} \mathrm{K}$ and $\lambda=635 \mathrm{~nm}$ at $93^{\circ} \mathrm{K}$. Rise in the magnitude of photocurrent at $93^{\circ} \mathrm{K}$ is observed in each case.

It is interesting to note that the doped thick films with lower copper concentration exhibit only a slight increase in the magnitude of photocurrent while films with higher $\mathrm{Cu}$ concentration $(0.3 \%$ by $\mathrm{wt})$ exhibit a drastic increase in the magnitude of photocurrent during these measurements at $93^{\circ} \mathrm{K}$.

The dependence of spectral response peak of these films on copper concentration is summarised in table 1 .

\section{Discussion}

It would be interesting to compare the results in the present investigation with those reported previously for single crystals, sintered layers and thin films. The values of photosensitivity of $\mathrm{CdS}$ photoconductors prepared by various conventional techniques are presented in table 2 which reveal the superior sensitivity of thick films.

It was anticipated that oxygen chemisorption could play an important role in the overall photoelectronic behaviour of our films. We have, therefore, carried out conductivity measurements during the oxygen adsorption-desorption cycles. 
Table 1. Effect of Cu concentration oin spectral resporse.

\begin{tabular}{ccc}
\hline $\begin{array}{c}\text { Concentration of } \\
\text { copper chloride } \\
\text { (wt \% of CdS) }\end{array}$ & \multicolumn{2}{c}{ Spectral response peak nm } \\
\cline { 2 - 3 } & At $300^{\circ} \mathrm{K}$ & At $93^{\circ} \mathrm{K}$ \\
\hline & & \\
Nil & $550-690$ & $510-520$ \\
$0 \cdot 05$ & $650-670$ & $660-680$ \\
$0 \cdot 10$ & $640-650$ & $630-640$ \\
$0 \cdot 15$ & $640-650$ & $630-640$ \\
$0 \cdot 20$ & $640-650$ & $630-640$ \\
$0 \cdot 25$ & $640-650$ & $630-640$ \\
$0 \cdot 30$ & $680-690$ & $640-650$ \\
$1 \cdot 00$ & $690-700$ & $640-650$ \\
\hline
\end{tabular}

Table 2. Photosensitivity values of CdS prepared by different techniques.

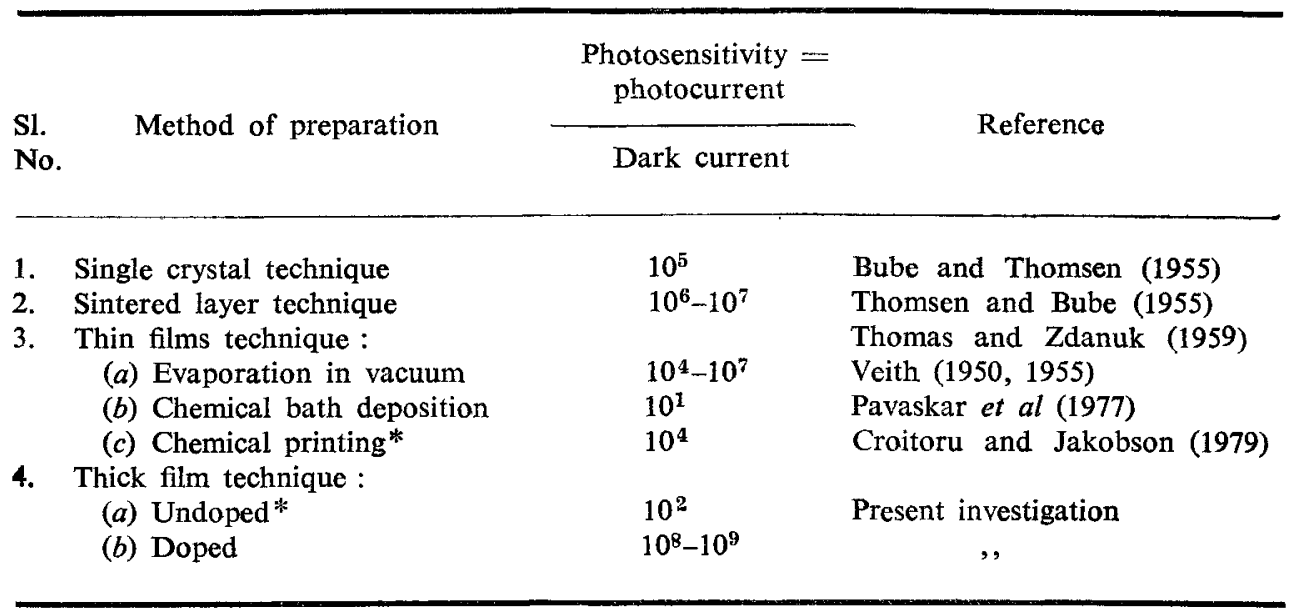

* These preparation techniques do not involve any impurity incorporation for sensitisation.

These cycles include (i) heating of the films upto $200^{\circ} \mathrm{C}$ in dark under vacuum, (ii) heating of these vacuun-treated films upto $200^{\circ} \mathrm{C}$ in dark and in air. We have observed that the room temperature dark conductivity changes by as much as 9 to 10 orders of magnitude as a result of heat treatment (i) and (ii) as against 6 to 7 orders change in the magnitude of dark conductivity as a result of such heat treatment reported earlier (Micheletti and Mark 1968). This leads to the conclusion that chemisorption of oxygen is more effective in lowering the dark current (while photocurrent remains unaltered) which ultimately improves photosensitivity in the present case. This perhaps could be related to the larger thickness $(\sim 20 \mu)$ and grain size $(\sim 4-10 \mu)$ in our films compared to the thickness $(\sim 3-5 \mu)$ and grain size $(\sim 1 \mu)$ for the films prepared by Micheletti and Mark (1968). This, along with the typical morphology of the surface, could have offered a high 
surface to volume ratio for the fused intergranular contacts or "necks" which are supposed to be responsible for ehemisorption in the sintered films. The possible role of grain structure in enhancing the photosensitivity is being studied using SEM and ESCA, the results of which will be published separately.

One distinct feature of the present results is the spectral sensitivity of the pure i.e. undoped CdS thick films at room temperature (figure 6, curve A). The shape of this curve appears to be different from that reported for undoped single crystals (Bube and Thomsen 1955) and thin films (Veith 1950; Pavaskar et al 1977). However, the spectral response associated with the same undoped sample at $93^{\circ} \mathrm{K}$ indicates a sharp response peak at $\lambda=515 \mathrm{~nm}$ (figure 6, curve B), which closely resembles in shape and response peak with the spectral response of pure CdS single crystals and thin films at room temperature. This may be attributed to the localised defect levels which lie in the forbidden gap just above the valence band. These levels are filled with electrons at room temperature. If transitions of electrons from these localised defect levels to conduction band are proposed, then such transitions would require relatively less excitation energy than that required for band to band transition. Therefore, the spectral response may extend to relatively longer wavelengths charactzristic of these defect excitation energies. The shape of the response curve in such a case will, of course, depends upon the actual transition probabilities. However, as the defect levels become empty at $93^{\circ} \mathrm{K}$ such electronic excitations are absent at this temperature. Hence, spectral response in this case corresponds to a wavelength which is characteristic of band to band tranisition. This response peak at $\lambda=515 \mathrm{~nm}$ corresponds to a value of $2 \cdot 4 \mathrm{eV}$. This is the generally accepted value for the band gap of CdS.

The highlights of the spectral response study made for the doped thick films (table 1) are : (i) there is a red shift in the spectral response peak with impurity incorporation. This observation is consistent with the work reported for doped single crystals (Woods 1957) sintered layers (Thomsen and Bube 1955; Thomas and Zdanuk 1959) and thin films of CdS (Veith 1950). When there is an appreciable concentration of compensated acceptor type of impurity, the possibility of direct electronic excitation from such impurity level lying in the forbidden gap to the conduction band is suggested by Rose (1963) and Bube (1967). Because of such impurity excitation, spectral response peak is displaced to the longer wavelength side. Further shift in response peak with increasing copper concentration could be attributed to a higher ratio of $\mathrm{Cu}$ to $\mathrm{Cl}$ centres (Thomas and Zdanuk 1959). This increase in $\mathrm{Cu}$ centres over $\mathrm{Cl}$ centres produces the observable deepening in the colour of the films. This change in body colour indicates a more efficient absorption of longer wavelengths (Thomas and Zdanuk 1959). An impurity banding which may occur as a result of increase in impurity density can also be expected to have some effect on this shift. (ii) It is observed that on lowering the temperature (a) there is no shift in response peak for the doped films with optimum copper concentration, (b) there is a negligible blue shift for the films with medium copper concentration, (c) there is an appreciable blue shift for the films with higher copper concentration. An appreciable shift in the band gap of CdS at low temperature is also presented by Lehman (1965) who studied the absorption edge as a function of impurity concentration for In doped CdS. He considered a tailing-off of the density of allowed states below the bottom edge of the 
conduction band because of crystal disorder. Our observations (c) in particular, could also be explained on similar lines.

Temperature dependence of the photocurrent as noticed during the spectral response measurements also forms an important feature of our study. Under the same conditions of spectral illumination, if the temperature is lowered from 300 to $93^{\circ} \mathrm{K}$, then (i) undoped films show a marked decrease in ph stocurrent (ii) highly doped films sh $\supset$ w a remarkable increase in the photocurrent and (iii) the medium doped films show a relatively little increase in the photocurrent with the varying copper concentration from $0.1 \%$ to $0.25 \%$ by wt. Observation (i) might be interpreted using the same model consisting of localised defect states as described earlier. These states when filled with electrons at room temperature possess high capture cross-section for photoexcited holes and small capture crosssection for photoexcited electrons. It means that these defect states behave as sensitising states at room temperature, therby exhibiting relatively high photoconductivity. But with the emptying of these states at $93^{\circ} \mathrm{K}$, they do not behave as sensitising states thereby exhibiting less photoconductivity. Owing to the complex nature of these defect states, the origin of which is still unknown to us, this model alone may not provide a satisfactory explanation. The observations (ii) and (iii) can be regarded as the consequence of high impurity concentration effects. It is well established by Bube and Dreeben (1959) that beyond a critical impurity concentration, the photosensitivity decreases rapidly at room temperature. Bube and others (Bube and Dreeben 1959; Bube et al 1962) have associated these effects to the decrease in the hole ionisation energy of the sensitising centres as a result of impurity banding. However, at low temperature quite a different type of situation arises in the case of highly doped films. There might be two possibilities: (i) Even with the decreased hole ionisation energy, thermal excitation of holes from the sensitising impurity states to valence band may not be possible at low temperature. (ii) Under the conditions of constant illumination, two steady-state Fermi-levels are shifted towards the respective band edges on lowering the temperature. As a result of this, more of the sensitising impurity states are embraced between the two Fermi levels. Thus, the conclusions (i) and (ii) imply that occupancy of the sensitising impurity states is governed more by recombination kinetics than by the thermal behaviour at $93^{\circ} \mathrm{K}$. This condition as proposed by Rose (1963) favours higher photoconductivity.

Apart from these basic studies, the usefulness of this fabrication technique to prepare highly sensitive photoconductor films may be emphasised. Further, the films prepared in this way have all the advantages of sintered photoconductors viz. superior sensitivity, large-area fabrication, high mechanical strength, uniformity and relative ease of impurity incorporation. In addition, there are certain other advanages which are inherent in the technique itself (Harper 1974).

\section{References}

Bube R H and Thomsen S M 1955 J. Chem. Phys. 2315

Bube R H and Dreeben A B 1959 Phys. Rev. 1151578

Bube R H, Lind E L and Dreeben A B 1962 Phys. Rev. 128532 
Bube R H 1967 Physics and Chemistry of II-VI Compounds (eds.) M Aven and J S Prener

(Amsterdam : North Holland Publishing Company) pp. 665-666

Croitoru N and Jakobson S 1979 Thin solid films 56 L5

Harper C A 1974 Hand-book of thick film hybrid micro-electronics (New York: Mc-Graw Hill Book Company)

Lehman W 1965 J. Electrochem. Soc. 1121150

Micheletti F B and Mark P 1968 J. Appl. Phys. 395274

Nicoll F H and Kazan B 1955 J. Opt. Soc. Am. 45647

Pavaskar N R, Menezes C A and Sinha A P B 1977 J. Electrochem. Soc. 124743

Rose A 1963 Concepts in Photoconductivity and allied problems (Interscience Publishers) p. 43

Sathaye S D and Sinha A P B 1976 Thin Solid Films 3715

Setty M S 1979 Indian J. Pure Appl. Phys. 17283

Thomas M J B and Zdanuk E J 1959 J. Electrochem. Soc. 106964.

Thomsen S M and Bube R H 1955 Rev. Sci. Instrum. 26664

Veith W 1950 Compt. Rend. 230947

Veith W $1955 Z$. Angew. Physik. 71

Wood J 1957 J. Electron Control 3225 